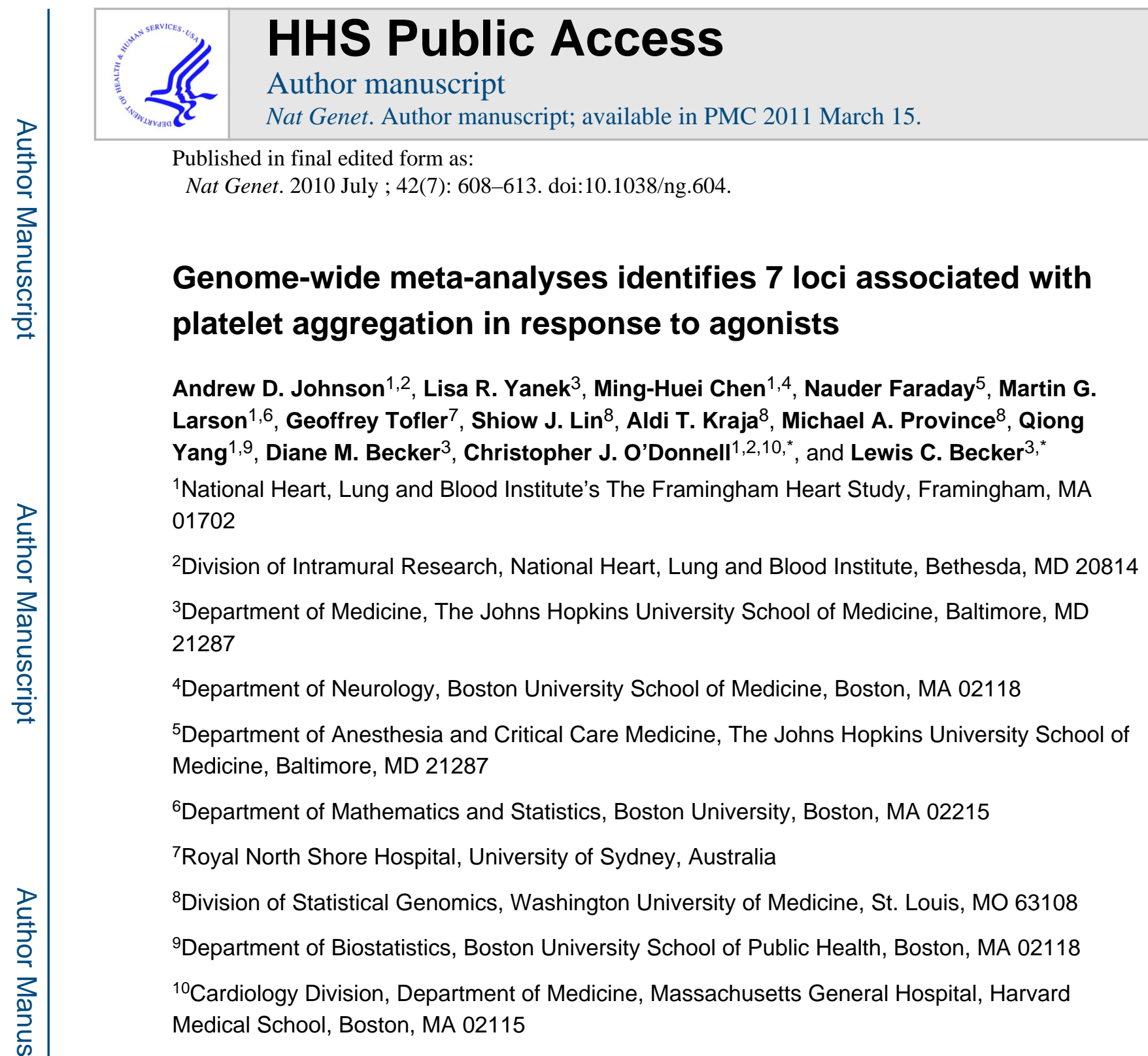

Platelet function mediates both beneficial and harmful effects on human health, but few genes are known to contribute to variability in the process. We tested association of 2.5 million SNPs with platelet aggregation responses to 3 agonists (ADP, epinephrine and collagen) in two European-ancestry cohorts ( $N \leq 2,753$ in the Framingham Heart Study, $N \leq$

\footnotetext{
Users may view, print, copy, download and text and data- mine the content in such documents, for the purposes of academic research, subject always to the full Conditions of use: http://www.nature.com/authors/editorial_policies/license.html\#terms

* these authors contributed equally

Database Accessions

HaemGen megakaryocyte results: ArrayExpress accession number E-TABM-633.

Author contributions

A.D. Johnson, L.R.Y., C.J.O and L.C.B. led the study. A.D. Johnson took primary responsibility for drafting the manuscript with contributions and editing from L.R.Y., M-H.C., N.F., M.G.L., G.T., A.T.K., Q.Y., D.M.B., C.J.O and L.C.B. G. Tofler, M.G.L. and C.J.O. were involved in the original guidance, collection and analysis of Framingham platelet phenotype data. L.R. Yanek, N.F., D.M.B. and L.C.B. were involved in the guidance, collection and analysis for the Genetic Study of Atherosclerosis Risk (GeneStar) phenotype data. S.J. Lin, A.T.K. and M.A.P. designed the database and analysis system used in GeneStar analyses. A.D. Johnson, L.R.Y., and M-H.C conducted genome-wide association analyses. A.D. Johnson, L.R.Y., M-H. C. and A.T.K. conducted additional analyses on SNP replication and the percent of variance explained. M-H. Chen conducted the meta-analyses. All authors read and approved the final version of the manuscript.
} 
1,238 in the Genetic Study of Atherosclerosis Risk), with replication $(P<0.05)$ in an African-American cohort ( $N \leq 840$ in the Genetic Study of Atherosclerosis Risk). We identified associations of seven loci with platelet aggregation, near/in $G P 6\left(P=4.6 \times 10^{-13}\right)$, PEARI $\left(P=3.4 \times 10^{-12}\right), A D R A 2 A\left(P=3.3 \times 10^{-11}\right)$, IK $3 C G\left(P=3.1 \times 10^{-9}\right), J M J D 1 C(P=$ $\left.1.6 \times 10^{-8}\right), \operatorname{MRVII}\left(P=2.0 \times 10^{-8}\right)$, and $\operatorname{SHH}\left(P=4.5 \times 10^{-8}\right)$. Evidence of replication was found for all loci. In total these findings provide new functional insights into platelet aggregation pathways and may suggest novel anti-platelet therapeutic targets.

Aggregation of blood platelets, a critical physiological response to vessel injury, leads to platelet thrombus formation and preserves vascular integrity. Responses are triggered by shear-stress or receptor agonists including ADP, collagen, epinephrine, and thrombin. Aggregation responses are mediated by glycoprotein receptors, and intracellular signaling pathways that trigger receptor activation and release of granules and thromboxane, further mediating feedback signaling. While normal platelet responsiveness maintains homeostasis and promotes wound healing, platelet hyperactivity may promote abnormal thrombosis, and the activation of platelets by plaques is a well-known contributor to acute coronary syndrome or stroke. Thus, several platelet aggregation mechanisms are targets for antiplatelet therapies for the treatment and prevention of cardiovascular disease1. Interindividual differences in anti-platelet responses suggest genetic variability, but studies to date, mainly focusing on candidate genes, have uncovered few consistent, replicated associations 2,3 .

The heritabilities of aggregation responses were previously established in the Framingham Heart Study (FHS) 4 and the Genetic Study of Atherosclerosis Risk (GS)5. Rare plateletrelated disorders are known6; however, with the exception of a limited scan in FHS7, no previous genome-wide scans have been reported for aggregation phenotypes. To investigate common genetic influences, we combined results from two cohorts' GWAS for platelet aggregation responses to three agonists: ADP, collagen, and epinephrine. Our meta-analysis includes European-ancestry (EA) study populations from FHS and GS, with participants free of symptoms of coronary artery disease and not taking anti-platelet medication. We conducted replication in an African-ancestry (AA) cohort that is also part of GS. The aim was to discover and replicate genome-wide significant loci associated with platelet aggregation and provide new insights into platelet aggregation mechanisms and its variability in humans.

The two GS cohorts were younger, and the GS AA sample had higher BMI and a higher prevalence of smoking, diabetes and hypertension than the two EA samples (Supplementary Table 1). There was no evidence for inflation of test statistics for the meta-analyses conducted, with all $\lambda \leq 1.01$. We observed 8 distinct platelet aggregation associations (Table 1) that met QC filters and surpassed a genome-wide significance threshold in meta-analysis $\left(P<5.0 \times 10^{-8}\right)$ with evidence for association in the same direction in both FHS and GS $(P$ $<0.05$ in both cohorts). Three regions were genome-wide significant for association with ADP-induced aggregation (Figure 1, Supplementary Figure 1a-c, Table 1): 1q23.1 (PEAR1 rs12566888 $\left.P=3.4 \times 10^{-12}\right), 11 \mathrm{p} 15.4\left(M R V I 1\right.$ rs7940646 $\left.P=2.0 \times 10^{-8}\right)$, and 7q36.3 ( $S H H$ rs2363910 $P=4.5 \times 10^{-8}$ ). The minor allele of the PEARI SNP was associated with a decrease in aggregation response, whereas the minor alleles of the $M R V I I$ and $7 \mathrm{q} 36.3$ 
variants were associated with increased responses (Table 2). All 3 regions showed evidence for replication $(P<0.05)$ in the African-ancestry sample based on genotyped SNPs that showed association with the same direction of effect (Table 3, PEARl rs12041331 $P=5.8 \times$ $10^{-9}$, MRVII rs1874445 $P=9.9 \times 10^{-3}$, SHH rs6943029 $\left.P=2.4 \times 10^{-3}\right)$. Three additional loci were modestly associated with increased aggregation responses to ADP in EA and also showed evidence for association in AA at $P<0.05$ (Supplementary Table 2).

Four regions had genome-wide significant associations for epinephrine-induced platelet aggregation (Figure 2, Supplementary Figure 1d-g, Table 1): 10q25.2 (ADRA2A rs4311994 $\left.P=3.3 \times 10^{-11}\right), 1 \mathrm{q} 23.1\left(P E A R 1 \mathrm{rs} 12566888 P=7.3 \times 10^{-10}\right), 7 \mathrm{q} 22.3(P I K 3 C G \mathrm{rs} 342286$ $\left.P=3.1 \times 10^{-9}\right)$, and 10q21.2 $\left(J M J D 1 C\right.$ rs10761741 $\left.P=1.6 \times 10^{-8}\right)$. For the ADRA2A, $P E A R 1$, and $P I K 3 C G$ regions, the minor alleles were associated with reduced epinephrineinduced aggregation (Table 2), while the minor allele of the peak SNP nearest JMJDIC was associated with increased aggregation (Table 2). Three of the regions showed consistent results in the African-ancestry sample (Table 3, PEARI rs12041331 $P=8.3 \times 10^{-17}$, $A D R A 2 A$ rs869244 $P=2.2 \times 10^{-6}, J M J D 1 C$ rs $\left.2893923 P=8.8 \times 10^{-3}\right)$. Four SNPs at $7 \mathrm{q} 22.3$ did not meet replication criteria (best SNP rs342296, $P=0.13$ ), though none was the peak SNP in EA meta-analyses. Eight additional regions showed moderate associations with epinephrine-induced aggregation in EA along with association in AA (Supplementary Table 2).

A single region, 19q13.42 (GP6 rs1671152, EA $P=4.6 \times 10^{-13}$, AA $P=0.048$ ), was associated with $\log _{10}$ collagen lag time response at a genome-wide significant level in FHS with $P=9.1 \times 10^{-14}$ (Supplementary Figure 1h, Supplementary Figure 2, Table 1). The peak associated SNP causes a Thr $>$ Lys change at amino acid 323. The strong association of the Lys allele with decreased collagen response (increased lag time, Table 2) observed in FHS was weakly replicated in GS (EA, $P=0.037$; AA, $P=0.048$ ). Our first meta-analysis compared collagen doses of $190 \mathrm{ug} / \mathrm{mL}$ (FHS, calf-skin-derived collagen) with $2 \mathrm{ug} / \mathrm{mL}$ (GS, equine-tendon-derived collagen) since these provided the most similar lag time distributions (Supplementary Figure 3), consistent with several orders of magnitude higher efficacy of calf- vs. equine-derived collagen (pers. comm., BioData, Inc.). We additionally analyzed associations of the single FHS dose compared with results from three other doses in GS $(1,5,10 \mathrm{ug} / \mathrm{mL})$, but did not find any additional genome-wide significant loci or gain stronger replication evidence for the GP6 locus. Three additional loci with evidence of moderate association in the main meta-analysis for collagen lag time in the EA sample showed similar association in the AA sample (Supplementary Table 2).

Given that the three platelet function agonists analyzed here target partially overlapping mechanisms of platelet aggregation, we inspected whether significantly associated loci overlapped across agonists. Four regions showed association with aggregation phenotypes in both the EA and AA samples and showed evidence for platelet responses to $\geq 2$ different agonists (Supplementary Table 3).

While an understanding of rare disorders of platelet aggregation has emerged6, the discovery of common genetic variations contributing to platelet aggregation has been marginally successful even though aggregation traits are heritable4,5. Prior studies were 
performed in modest sample sizes, utilized candidate gene approaches focusing on glycoprotein receptors, and often employed variable conditions in diseased populations. By adopting a GWAS approach in large cohorts of relatively healthy individuals and using similar platelet-rich plasma (PRP)-derived aggregation phenotypes, we discovered or replicated strong associations $\left(P=5.0 \times 10^{-8}\right)$ for 7 distinct loci with platelet aggregation, and found suggestive evidence for many additional loci (summarized in Table 4 and Supplementary Table 4). The findings for the PEAR18,9, ADRA2A10,11 and GP612,13 regions provide strong evidence in a much larger sample than past studies, while the associations in the regions of MRVII, SHH, JMJDIC, and PIK3CG are novel.

Platelet endothelial aggregation receptor-1 (PEARI) undergoes tyrosine phosphorylation after platelet-platelet contact14. A PEAR1 promoter region variant (rs2768759) was associated with increased aggregation in PRP, most strongly in response to epinephrine, and in both pre- and post-aspirin treatment conditions8. Recently a candidate gene study found association of PEARI SNPs with ADP and collagen responses in 500 whole blood-derived samples, and an increase in surface PEAR1 expression upon activation9. These candidate gene studies8,9 had limited coverage of the PEAR1 region. In our study, the prior SNPs8,9 were not among the strongest associations; instead, the peak associations with ADP and epinephrine response lie within a relatively conserved region of intron 1 of PEARI.

Variation in ADRA2A receptor numbers and polymorphisms in ADRA2A that influence epinephrine-induced aggregation in diverse populations were reported nearly 15 years ago10,11. The association of ADRA2A expression with epinephrine response is logical, given that ADRA2A serves as the primary receptor for epinephrine on platelets. Additional reports in small samples have reproduced $A D R A 2 A$ associations 15 , including recognition of complex population patterns in the region and effects on RNA levels in vitro16. Notably, unlike prior studies focused on the immediate gene region, the peak SNP associations we observed are somewhat distant and 3' from the gene (EA, rs4311994, 63kb, $P=3.3 \times 10^{-11}$; AA, rs869244, $70 \mathrm{~kb}, P=2.2 \times 10^{-6}$ ) suggesting partial LD with causal variants close to the gene or possible long range regulatory elements.

The association of GP6 variants with collagen lag time is biologically plausible, as GP6 is the primary glycoprotein receptor that mediates collagen responses in platelets. The peak GP6 SNP in FHS, a nonsynonymous variant (Thr323Lys), was strongly associated with collagen lag time (rs1671152, $\left.P=9.1 \times 10^{-14}\right)$. Notably rs 1671152 is in LD with rs 1613662 (Ser219Pro, HapMap CEU ${ }^{2}=1.0$ ). Both variants have been associated with diminished collagen expression or downstream responses (e.g.,13,17). Due to multiple GP6 protein isoforms formed by splicing and a frameshift, Thr323Lys is alternatively His322Aln in a shorter isoform. Five nSNPs are in LD, including Ser219Pro and Thr323Lys/His322Aln, making it difficult to determine which are functional13,17, although a recent study supports an effect on receptor binding of Thr323Lys/His322Aln within this haplotype17. GP6 plays a role in thrombus formation18. Interestingly, two studies recently replicated association of the 219Pro allele with reduced risk for deep vein thrombosis, indicating potential clinical relevance for genetic findings in GP619,20. In our study, both Thr323Lys and Ser219Pro were similarly associated with collagen lag time (EA, $P=4.6 \times 10^{-13}$ vs. $P=4.7 \times 10^{-12}$, AA, $P=0.048$ vs. $P=0.08$ ). 
MRVII (also known as IRAG), which showed both ADP- and epinephrine-induced associations (Table 3, Supplementary Table 3), has prior evidence of functions in platelet aggregation. MRVI1 is a member of a signaling complex which influences smooth muscle cell relaxation through negative regulation of INP3-induced calcium signaling21. In mice MRVI1 plays a direct role in the inhibition of platelet aggregation and in vivo thrombosis22. There is also prior evidence for platelet-related functions for some genes at other novel loci we report. In a human heterologous system $\mathrm{SHH}^{+}$microvesicles induce differentiation along a megakaryocyte lineage suggesting a link to platelet biology23. Polymorphisms near PIK3CG (rs342293) were recently associated with decreased mean platelet volumes 24 . The SNP rs342286, associated here with epinephrine-induced aggregation $\left(P<3.1 \times 10^{-9}\right)$, is in

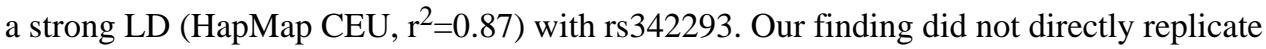
in African-Americans $(P=0.13)$ although the direction of effect was similar. A direct function for PIK3CG in platelet signaling has been demonstrated25, making it a putative mediator of the effects observed in ours and the prior study 24 .

We note associations near two genes, MRVII and PIK3CG, with prior known platelet functions which are related to intracellular signaling pathways in platelets21,22,24,25. When we attempted to replicate regions with modest evidence for association $\left(P<1.0 \times 10^{-4}\right.$ in EA) we found further evidence for regions that encode proteins with known involvement in platelet signaling pathways, including RGS1826-29, RAP1B30-33, and RAPGEF234,35, as well as others with putative platelet functions including ST3GAL436,37 and PRNP38,39 (Supplementary Table 2, Supplementary Table 4). Evaluation of prior candidate SNPs and gene regions from the literature indicated evidence consistent with prior studies in the regions of $P 2 R Y 12$, a receptor that mediates ADP responses, TAOKI, previously associated with mean platelet volume 40 , and FCERIG, previously associated with collagen response9 and here associated with collagen lag time (Supplementary Table 5).

Combining our novel findings with prior studies, 7 loci are strongly implicated for genetic roles in platelet aggregation, with several more loci having consistent evidence in $\geq 2$ populations (Supplemental Table 4). These additional loci contain genes with compelling biological links to platelet function and warrant further investigation. Known functions of genes at the novel loci suggest that in addition to glycoprotein receptors, proteins involved in intracellular signal transduction pathways and platelet homeostasis are also critical to mediating aggregation responses. Some variants from our study (e.g., GP6) have already been associated with clinically apparent cardiovascular disease. It will be important to conduct further functional and clinical studies to examine the clinically relevant function of genetic variants in these loci and the potential of corresponding proteins as targets for drug treatment, given the central role of platelet function in multiple disease etiologies including thrombosis, myocardial infarction, stroke, wound healing and response to infection.

\section{Platelet aggregation phenotype collection}

FHS is a community-based, prospective, longitudinal study following 3 generations of participants. The Offspring cohort studied here represents the second generation, including spouses44. GS is a family-based, prospective study. Apparently healthy subjects free of current aspirin or anti-platelet use were included in phenotype collection for FHS4,45 and 
GS8,46. FHS participants were excluded from analysis if they self-reported use of aspirin or anti-platelet medication, or in the absence of response to $5 \mathrm{mg} / \mathrm{mL}$ arachidonic acid which was presumed indicative of aspirin therapy4,45. GS families were identified from probands with documented premature (age $<60)$ coronary artery disease (CAD) in one of ten Baltimore area hospitals; unaffected, apparently healthy siblings, offspring of the siblings and probands, and parents of the offspring were recruited from 2003-2006 for a study of platelet reactivity 8,46 . Eligible participants were free of coronary artery disease, had no history of any bleeding disorder or hemorrhagic event, and no serious comorbidities. Participants with a history of aspirin intolerance, abnormal platelet count, hematocrit, or white blood cell count, or current use of anticoagulants or antiplatelet agents were excluded. Use of aspirin and/or nonsteroidal anti-inflammatory drugs was prohibited for ten days before the study visit.

Both studies isolated platelet-rich plasma (PRP) from blood samples taken from participants after fasting, and measured platelet aggregation after addition of agonists using a fourchannel aggregometer (BioData Corp., Horsham, PA). FHS samples were collected at exam cycle 5 from participants' antecubital vein while in the supine position between $8 \mathrm{AM}$ and 9AM (previously described) 4,45 . Blood was placed in a $3.8 \%$ sodium citrate solution and centrifuged at $160 \times \mathrm{g}$ for $5 \mathrm{~min}$ at room temperature to separate out platelet-rich plasma (PRP). In GS participants, after an 8-12 h fast, blood was drawn and collected in vacutainer tubes containing sodium citrate (3.2\%), after discarding the first $4 \mathrm{ml}$. PRP was prepared by centrifugation of whole blood at $180 \times \mathrm{g}$ for $15 \mathrm{~min}$, and platelet poor plasma (PPP) was prepared by centrifugation at $2000 \times \mathrm{g}$ for $10 \mathrm{~min}$. PRP was diluted with PPP to adjust platelet counts to 200,000/ul. All GS platelet function studies were completed within $2 \mathrm{~h}$ after the blood draw.

FHS tested aggregation for periods 4 min post-ADP $(0.05,0.1,0.5,1.0,3.0,5.0,10.0,15.0$ $u \mathrm{M}), 5$ min post-epinephrine $(0.01,0.03,0.05,0.1,0.5,1.0,3.0,5.0,10.0 u \mathrm{M})$, and lag time (s) to aggregation with $190 \mathrm{ug} / \mathrm{mL}$ calf-skin-derived Type-I collagen (BioData Corp., Horsham, PA). Threshold concentrations (EC50) were determined as the minimal concentration of agonist required to produce a $>50 \%$ aggregation. Testing was not conducted at higher concentrations if $>50 \%$ aggregation was observed. The maximal aggregation response (\% aggregation) was also determined for each participant at each concentration tested. GS recorded maximal aggregation (\% aggregation) for periods $5 \mathrm{~min}$ post-ADP $(2.0,10.0 u \mathrm{M})$ and post-epinephrine $(2.0,10.0 u \mathrm{M})$, and lag time (s) to aggregation with equine-tendon-derived Type-I collagen $(1,2,5$ and $10 \mathrm{ug} / \mathrm{mL}$, Chronolog Corp., Havertown, PA).

\section{Genotyping and imputation}

DNA was extracted and genotyped for consenting FHS participants with the Affymetrix 500K array and an additional gene-focused 50K array as part of the SNP Health Association Resource (SHARe) project. DNA was extracted and genotyped for the GS samples with the Illumina 1M (duo) array at deCODE Genetics (Reykjavik, Iceland). FHS and GS both used MACH to impute 2.54 million SNPs based on the HapMap CEU phased haplotypes (release 22). SNPs were excluded from imputation in FHS that had MAF $<1 \%$, HWE $P<$ 
$1.0 \times 10^{-6}$, SNP call rate $<97.0 \%$, MISHAP test $P<1.0 \times 10^{-9}$, Mendelian errors $>100$, or were missing from the HapMap CEU population release 22. Two hundred unrelated individuals were selected from FHS who had low SNP missingness, low numbers of Mendelian errors and who did not show up as outliers in EIGENSTRAT 2.047 (default parameters). The 200 individuals were used to infer MACH model parameters first (MACH flags used: --rounds 100 -greedy), and subsequently applied on all 8,481 individuals (MACH flags used: --greedy --mle -crossovermap -errormap). FHS samples were excluded from GWAS analysis if they had genome-wide call rates $<97.0 \%$, high Mendelian error rates or exhibited genome-wide heterozygosity $>5$ s.d. away from the mean. In GS, participants with sex discrepancies or Mendelian errors $>2 \%$ were excluded from imputation. SNPs excluded from imputation had MAF $<1 \%$, HWE $P<1.0 \times 10^{-6}$, or call rate $<95.0 \%$. GS selected 200 EA individuals (pre-screened to be unrelated) by prioritizing those individuals with low missingness, balanced in number of males and females; none of them identified as outliers by EIGENSTRAT 2.0. Similar to FHS, the 200 pre-selected individuals were used to infer model parameters first (MACH flags used: --rounds 100 -greedy), and subsequently that model was applied to all 1,991 EA individuals with genotypes (MACH flags used: --greedy --mle -crossovermap --errormap).

\section{Genetic analyses in each cohort}

Both cohorts evaluated age- and sex- adjusted models for aggregation phenotypes. FHS and GS included the principal components (PC) from EIGENSTRAT 2.047 ( $\mathrm{n}=8$ and $\mathrm{n}=2$, respectively) as covariates to account for potential population admixture. Collagen lag times and EC50 concentrations were $\log _{10}$ transformed before analysis. Epinephrine maximal aggregations were BLOM-transformed due to non-Gaussian distributions. Linear mixed effects (LME) models were used in FHS and GS individually to test the association under an additive model between a SNP and specific phenotype adjusted for age, sex and PCs. The linear mixed effects (LME) model follows in a matrix form: $\boldsymbol{Y}=\boldsymbol{X} \boldsymbol{B}+\boldsymbol{Z} \boldsymbol{U}+\varepsilon$, where $\boldsymbol{Y}$ is an $m \times 1$ vector of responses; $\boldsymbol{X}$ is an $m \times p$ design matrix of the fixed effects; $\boldsymbol{B}$ is the parameter $p \times 1$ vector of fixed effects; $\boldsymbol{Z}$ is an $m \times q$ incidence matrix of random effects, and $\boldsymbol{U}$ is a $q \times 1$ vector of random effects with $E(\boldsymbol{U})=\mathbf{0}$, and covariance matrix $\boldsymbol{G} ; \boldsymbol{O}$ is an $m$ $\times 1$ vector of random effects with $E(\boldsymbol{0})=\mathbf{0}$ and covariance matrix $\boldsymbol{R}$. In the fixed effects we included SNP genotypes using an additive model ( 0 for one major allele, 1 for the heterozygote, and 2 for the minor allele homozygote genotype) for the original genotypes and dosage (probabilistic estimations) for the imputed genotypes. We tested whether the SNP additive effects differed from zero. FHS used the R kinship and GWAF packages48, accounting for familial relatedness, while GS used PROC MIXED in SAS (v. 9.1.3 for Linux OS) with the option for EMPIRICAL variance49 and including the family identification number in the random effects to account for relatedness.

The agonist conditions and number of subjects analyzed in GWAS were as follows: for ADP: in FHS, EC50 ( $\mathrm{n}=2,372), 3 u \mathrm{M}(\mathrm{n}=2,753), 5 u \mathrm{M}(\mathrm{n}=1,803)$ and in GS, $2 u \mathrm{M}(\mathrm{n}=1,110)$, $10 u \mathrm{M}(\mathrm{n}=1,227)$; for epinephrine: in FHS, EC50 ( $\mathrm{n}=2,364), 1 u \mathrm{M}(\mathrm{n}=2,166), 3 u \mathrm{M}(\mathrm{n}=1,220)$, and for GS, $2 u \mathrm{M}(\mathrm{n}=1,238), 10 u \mathrm{M}(\mathrm{n}=1,232)$; for collagen lag time: in FHS $(\mathrm{n}=2,310)$ and in GS, 1, 2, 5 and $10 \mathrm{ug} / \mathrm{mL}$ (n=931, 1,162, 1,222, 1,223, respectively). In GS, participants from 230 families with African-ancestry were used in replication analyses: collagen lag 2 
$u \mathrm{~g} / \mathrm{mL}(\mathrm{n}=763), \operatorname{ADP} 2 u \mathrm{M}(\mathrm{n}=788)$ and $10 u \mathrm{M}(\mathrm{n}=836)$, epinephrine $2 u \mathrm{M}(\mathrm{n}=837)$ and $10 u \mathrm{M}(\mathrm{n}=840)$.

\section{Meta-analysis}

SNPs considered in the meta-analyses did not have missing information for either cohort, and had MAF $>=1.0 \%$ and an imputation observed to expected ratio $>=0.30$ in both cohorts. After this QC filtering 2.33 million SNPs were included in the meta-analysis for each trait. Sample-size weighted meta-analysis was conducted with the software METAL combining the GS and FHS. The phenotypes used in meta-analyses were for the same agonists at the concentrations with the best available overlap (see Supplementary Table 1). Additionally, when meta-analyzing FHS threshold response (EC50) associations for ADP and epinephrine and GS maximal aggregation, the sign of the beta in FHS was flipped, since threshold response and maximal aggregation are inversely related. Results presented are based on individual cohort age-, sex- and PC-adjusted analyses, and meta-analyses corrected for individual study genomic control inflation rates. Regional association plots (Supplementary Figure 1a-h) were generated with SNAP41.

\section{Replication analysis}

We conducted testing for replication in an independent, African-ancestry sample within GS. Since LD patterns in general for African-ancestry individuals at the genome level are more complex and diverse than in populations that are primarily of European-ancestry, relying on single sentinel SNPs from European-ancestry individuals or on imputed or proxy SNPs in African-ancestry individuals for replication comparisons could lead to spurious associations. Thus, we chose to focus replication efforts on all SNPs in regions with evidence for association in the EA meta-analyses $\left(P<1.0 \times 10^{-4}\right)$ that were directly genotyped with the Illumina $1 \mathrm{M}$ (duo) array and had MAF $\geq 1.0 \%$ in the AA replication sample. We searched for evidence of age- and sex-adjusted association in the AA samples only for the same platelet aggregation phenotypes corresponding to those in the main scan. Replication evidence was defined by SNPs with effects in the same direction in AA samples as in EA samples at a $P<0.05$ threshold.

\section{Supplementary Material}

Refer to Web version on PubMed Central for supplementary material.

\section{Acknowledgements}

This work was supported by the National Heart, Lung and Blood Institute's Framingham Heart Study (Contract No. N01-HC-25195) and its contract with Affymetrix, Inc for genotyping services (Contract No. N02-HL-6-4278). This research was conducted in part using data and resources from the Framingham Heart Study of the National Heart Lung and Blood Institute of the National Institutes of Health and Boston University School of Medicine. The analyses reflect intellectual input and resource development from the Framingham Heart Study investigators participating in the SNP Health Association Resource (SHARe) project. A portion of this research utilized the Linux Cluster for Genetic Analysis (LinGA-II) funded by the Robert Dawson Evans Endowment of the Department of Medicine at Boston University School of Medicine and Boston Medical Center. This work was supported by the National Heart, Lung and Blood Institute through the PROGENI (U01 HL72518) and STAMPEED (R01 HL087698-01) consortia, and through R01-HL-48157. This research was conducted in part using the resources of the Johns Hopkins General Clinical Research Center, funded through the National Center for Research Resources, 
M01-RR000052, and the Washington University DSG cluster. We thank Georg Ehret and Santhi Ganesh for providing R code that was modified to generate plots displayed in Figures 1-3.

\section{References}

1. Wolff T, Miller T, Ko S. Aspirin for the primary prevention of cardiovascular events: an update of the evidence for the U.S. Preventive Services Task Force. Ann. Intern. Med. 2009; 150:405-410. [PubMed: 19293073]

2. Quinn MJ, Topol EJ. Common variations in platelet glycoproteins: pharmacogenomic implications. Pharmacogenomics. 2001; 2:341-352. [PubMed: 11722284]

3. Faraday N, Becker DM, Becker LC. Pharmacogenomics of platelet responsiveness to aspirin. Pharmacogenomics. 2007; 8:1413-1425. [PubMed: 17979514]

4. O'Donnell CJ, et al. Genetic and environmental contributions to platelet aggregation: the Framingham Heart Study. Circ. 2001; 103:3051-3056.

5. Bray PF, et al. Heritability of platelet function in families with premature coronary artery disease. J. Thromb. Haemo. 2007; 5:1617-1623.

6. Salles II, et al. Inherited traits affecting platelet function. Blood Rev. 2008; 22:155-172. [PubMed: 18180086]

7. Yang Q, Kathiresan S, Lin JP, Tofler GH, O'Donnell CJ. Genome-wide association and linkage analyses of hemostatic factors and hematological phenotypes in the Framingham Heart Study. BMC Med. Gen. 2007; 8:S12.

8. Herrera-Galeano JE, et al. A novel variant in the platelet endothelial aggregation receptor-1 gene is associated with increased platelet aggregability. Arterioscler. Thromb. Vasc. Biol. 2008; 28:14841490. [PubMed: 18511696]

9. Jones CI, et al. on behalf of the Bloodomics consortium. A functional genomics approach reveals novel quantitative trait loci associated with platelet signaling pathways. Blood. 2009 May 8. published online.

10. Freeman K, et al. Genetic polymorphism of the $a_{2}$-adrenergic receptor is associated with increased platelet aggregation, baroreceptor sensitivity, and salt excretion in normotensive humans. Amer. J. Hyper. 1995; 8:863-869.

11. Kambayashi J, et al. Prevalence of impaired responsiveness to epinephrine in platelets among Japanese. Thromb. Res. 1996; 81:85-90. [PubMed: 8747523]

12. Croft SA, et al. Novel platelet membrane glycoprotein VI dimorphism is a risk factor for myocardial infarction. Circ. 2001; 104:1459-1463.

13. Joutsi-Korhonen L, et al. The low-frequency allele of the platelet collagen signaling receptor glycoprotein VI is associated with reduced functional responses and expression. Blood. 2003; 101:4372-4379. [PubMed: 12560230]

14. Nanda N, et al. Platelet endothelial aggregation receptor 1 (PEAR1), a novel epidermal growth factor repeat-containing transmembrane receptor, participates in platelet contact-induced activation. JBC. 2005; 280:24680-24689.

15. Yabe M, et al. Identification of ADRA2A polymorphisms related to shear-mediated platelet function. Biochem. Biophys. Res. Comm. 2006; 347:1001-1005. [PubMed: 16854373]

16. Small KM, Brown KM, Seman CA, Theiss CT, Liggett SB. Complex haplotypes derived from noncoding polymorphisms of the intronless $\mathrm{a}_{2 \mathrm{~A}}$-adrenergic gene diversify receptor expression. PNAS. 2006; 103:5472-5477. [PubMed: 16567612]

17. Trifiro E, et al. The low-frequency isoform of platelet glycoprotein VIb attenuates ligand-mediated signal transduction but not receptor expression or ligand binding. Blood. 114:1893-1899. [PubMed: 19465689]

18. Massberg S, et al. A crucial role of glycoprotein VI for platelet recruitment to the injured arterial wall in vivo. J. Exp. Med. 2003; 197:41-49. [PubMed: 12515812]

19. Bezemer ID, et al. Gene variants associated with deep vein thrombosis. JAMA. 2008; 299:13061314. [PubMed: 18349091]

20. Tregouet DA, et al. Common susceptibility allele are unlikely to contribute as strongly as the $F V$ and $A B O$ loci to VTE risk: results from a GWAS approach. Blood. 2009 Mar 10. published online. 
21. Schlossmann J, et al. Regulation of intracellular calcium by a signaling complex of IRAG, IP3 receptor and cGMP kinase Ibeta. Nature. 2000; 404:197-201. [PubMed: 10724174]

22. Antl M, et al. IRAG mediates NO/cGMP-dependent inhibition of platelet aggregation and thrombus formation. Blood. 2007; 109:552-559. [PubMed: 16990611]

23. Martinez MC, et al. Transfer of differentiation signal by membrane microvesicles harboring hedgehog morphogens. Blood. 2006; 108:3012-3020. [PubMed: 16778137]

24. Soranzo N, et al. A novel variant on chromosome 7q22.3 associated with mean platelet volume, counts, and function. Blood. 2009; 113:3831-3837. [PubMed: 19221038]

25. Schoenwaelder SM, et al. Identification of a unique co-operative phosphoinositide 3-kinase signaling mechanism regulating integrin $\alpha_{\mathrm{IIb}} \beta_{3}$ adhesive function in platelets. J. Biol. Chem. 2007; 282:28648-28658. [PubMed: 17673465]

26. Yowe D, et al. RGS18 is a myeloerythroid lineage-specific regulator of G-protein-signaling molecule highly expressed in megakaryocytes. Biochem. J. 2001; 359:109-118. [PubMed: 11563974]

27. Gagnon AW, Murray DL, Leadley RJ. Cloning and characterization of a novel regulator of G protein signaling in human platelets. Cell Signal. 2002; 14:595-606. [PubMed: 11955952]

28. Kim SD, et al. The expression patterns of RGS transcripts in platelets. Platelets. 2006; 17:493-497. [PubMed: 17074726]

29. Garcia A, et al. Differential proteome analysis of TRAP-activated platelets: involvement of DOK-2 and phosphorylation of RGS proteins. Blood. 2004; 103:2088-2095. [PubMed: 14645010]

30. Guidetti GF, et al. The $\mathrm{G}_{\mathrm{i}}$-coupled $\mathrm{P} 2 \mathrm{Y} 12$ receptor regulates diacylglycerol-mediated signaling in human platelets. J. Biol. Chem. 2008; 283:28795-28805. [PubMed: 18755689]

31. Cannobbio I, et al. Genetic evidence for a predominant role of PI3K $\beta$ catalytic activity in ITAMand integrin-mediated signaling in platelets. Blood. 2009 Jun 10. published online.

32. Wang Z, et al. Rap1b is critical for glycoprotein VI-mediated but not ADP receptor-mediated $\alpha_{2} \beta_{1}$ activation. J. Thromb. Haem. 2009; 7:693-700.

33. Chrzanowska-Wodnicka $M$, et al. Rap $1 \mathrm{~b}$ is required for normal platelet function and hemostasis in mice. J. Clin. Invest. 2005; 115:680-687. [PubMed: 15696195]

34. Letschka T, et al. PKC- $\theta$ selectivity controls the adhesion-stimulating molecule Rap1. Blood. 2008; 112:4617-4627. [PubMed: 18796635]

35. Severson EA, Lee WY, Capaldo CT, Nusrat A, Parkos CA. Junctional adhesion molecule A interacts with Afadin and PDZ-GEF2 to activate Rap1a, regulate $\beta 1$ integrin levels, and enhance cell migration. Mol. Biol. Cell. 2009; 20:1916-1925. [PubMed: 19176753]

36. Ellies LG, et al. Sialyltransferase ST3Gal-IV operates as a dominant modifier of hemostasis by concealing asialoglycoprotein receptor ligands. PNAS. 2002; 99:10042-10047. [PubMed: 12097641]

37. Grewal PK, et al. The Ashwell receptor mitigates the lethal coagulopathy of sepsis. Nat. Med. 2008; 14:648-655. [PubMed: 18488037]

38. Holada K, Glierova H, Simak J, Vostal JG. Expression of cellular prion protein on platelets from patients with gray platelet or Hermansky-Pudlak syndrome and the protein's association with agranules. Haematologica. 2006; 91:1126-1129. [PubMed: 16885055]

39. Jones M, et al. Human platelets as a substrate source for the in vitro amplification of the abnormal prion protein $\left(\mathrm{PrP}^{\mathrm{Sc}}\right)$ associated with variant Creutzfeldt-Jakob disease. Transfusion. 2009; 49:376-384. [PubMed: 18980616]

40. Meisinger $\mathrm{C}$, et al. A genome-wide association study identifies three loci associated with mean platelet volume. Am. J. Hum. Genet. 2009; 84:66-71. [PubMed: 19110211]

41. Johnson AD, et al. SNAP: a web-based tool for identification and annotation of proxy SNPs using HapMap. Bioinformatics. 2008; 24:2938-2939. [PubMed: 18974171]

42. Shaughnessy JD, et al. Mrvil, a common MRV integration site in BXH2 myeloid leukemias, encodes a protein with homology to a lymphoid-restricted membrane protein Jaw1. Oncogene. 18:2069-2084. [PubMed: 10321731]

43. Watkins NA, et al. A HaemAtlas: characterizing gene expression in differentiated human blood cells. Blood. 2009; 113:1-9. 
44. Kannel WB, Feinleib M, McNamara PM, Garrison RJ, Castelli WP. An investigation of coronary heart disease in families: the Framingham Offspring Study. Am. J. Epidemiol. 1979; 110:281-290. [PubMed: 474565]

45. Mukamal KJ, et al. Alcohol consumption and platelet activation and aggregation among women and men: The Framingham Offspring Study. Alc. Clin. Exp. Res. 2005; 29:1906-1912.

46. Becker DM, et al. Sex differences in platelet reactivity and response to low-dose aspirin therapy. JAMA. 2006; 295:1420-1427. [PubMed: 16551714]

47. Price AL, et al. Principal components analysis corrects for stratification in genome-wide association studies. Nat. Gen. 2006; 38:904-909.

48. Chen MH, Yang Q. GWAF: an R package for genome-wide association analysis with family data. Bioinformatics. 2009 Dec 29. published online.

49. Littell, RC.; Milliken, GA.; Stroup, WW.; Wolfinger, RD. SAS System for Mixed Models. Cary, North Carolina: SAS Institute; 1996. 


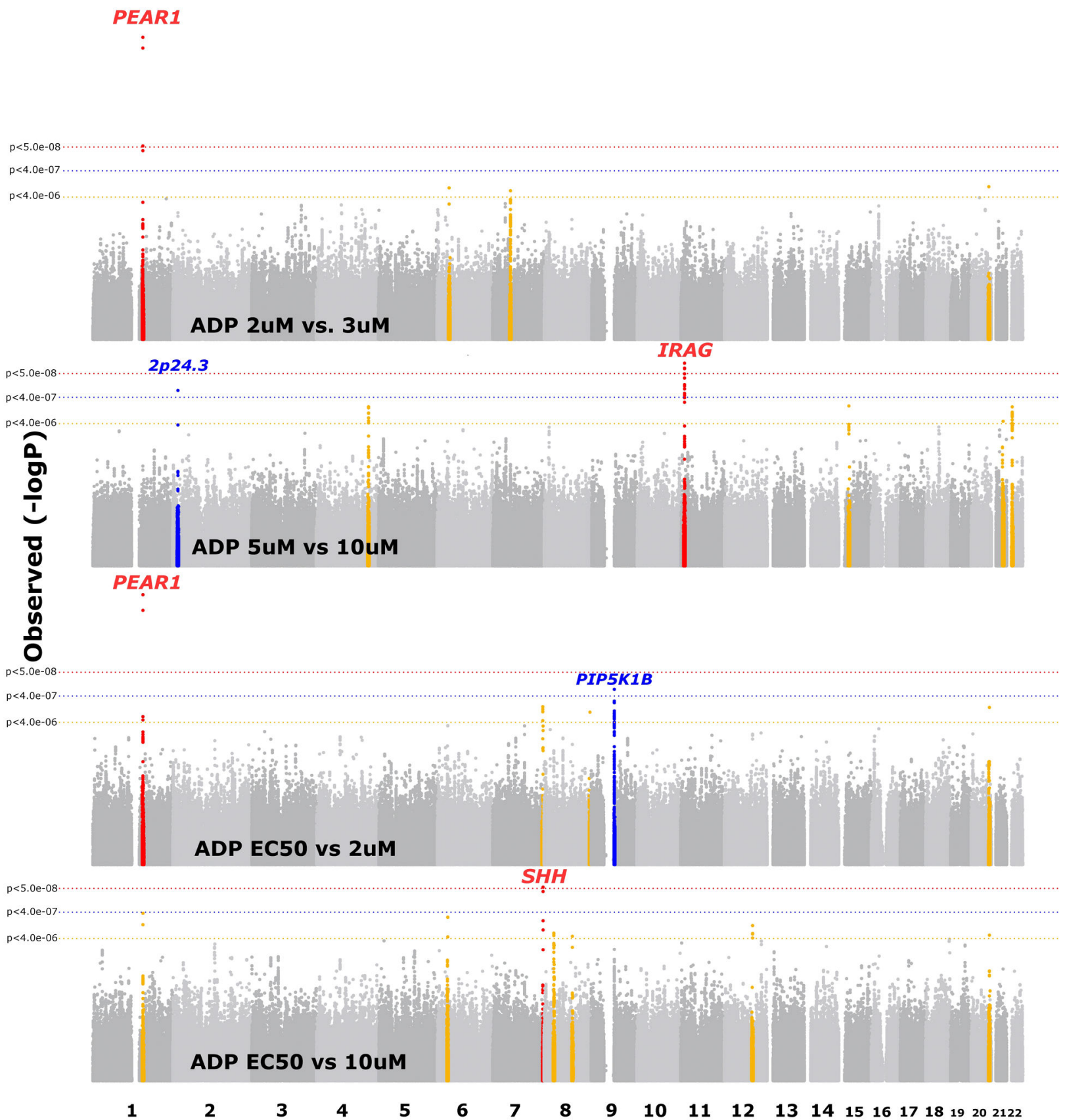

Figure 1. 


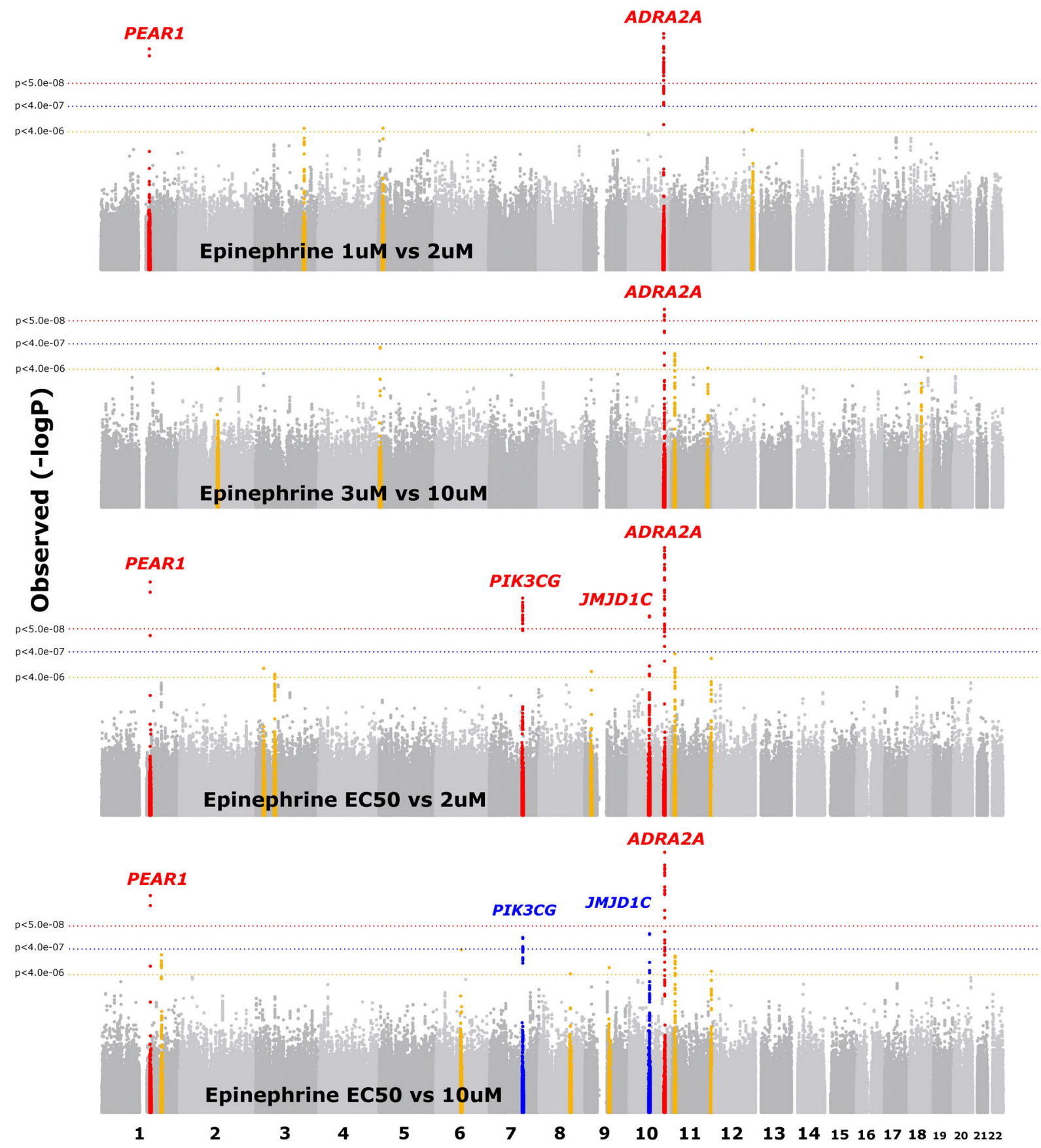

Figure 2. 


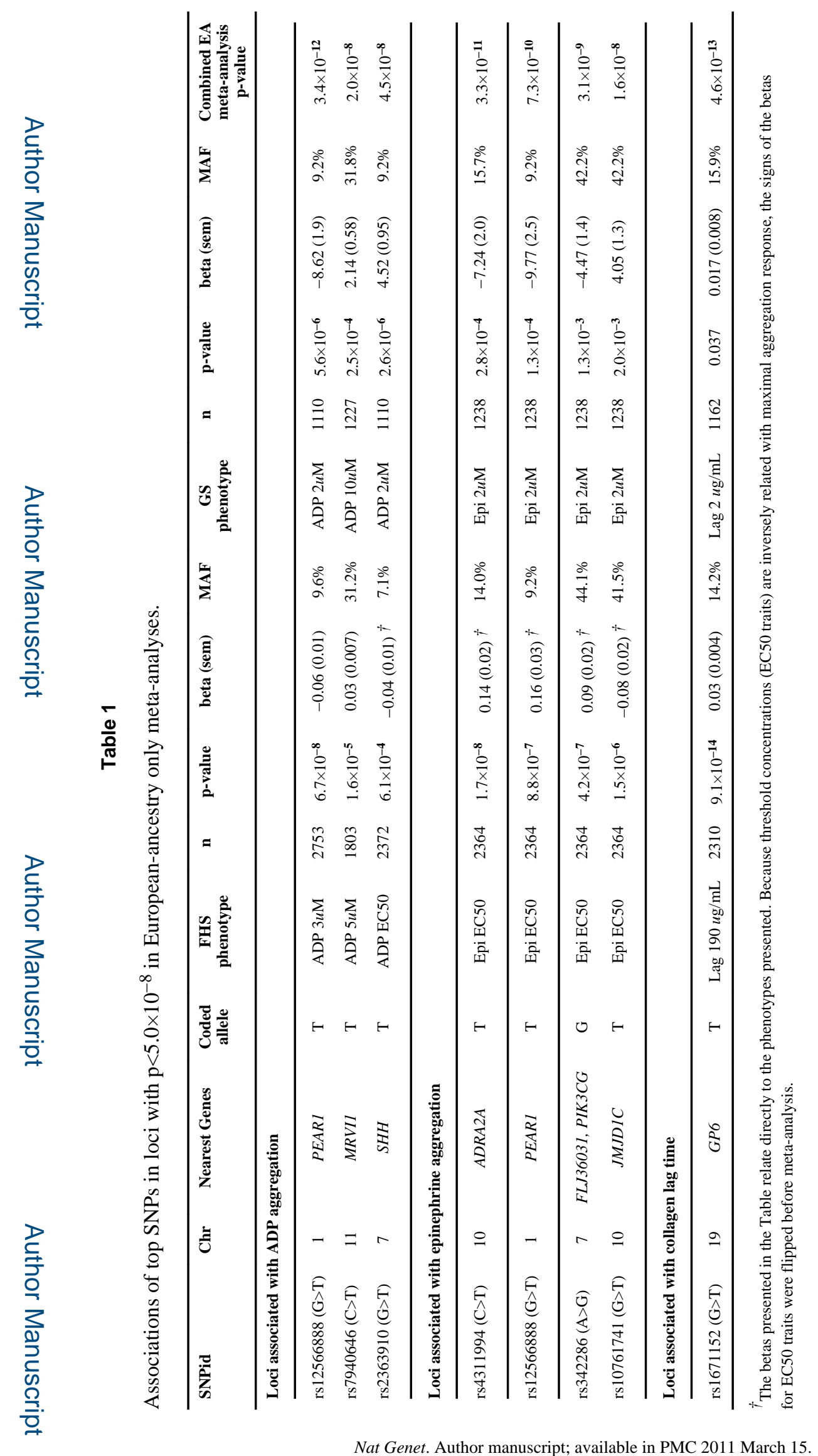




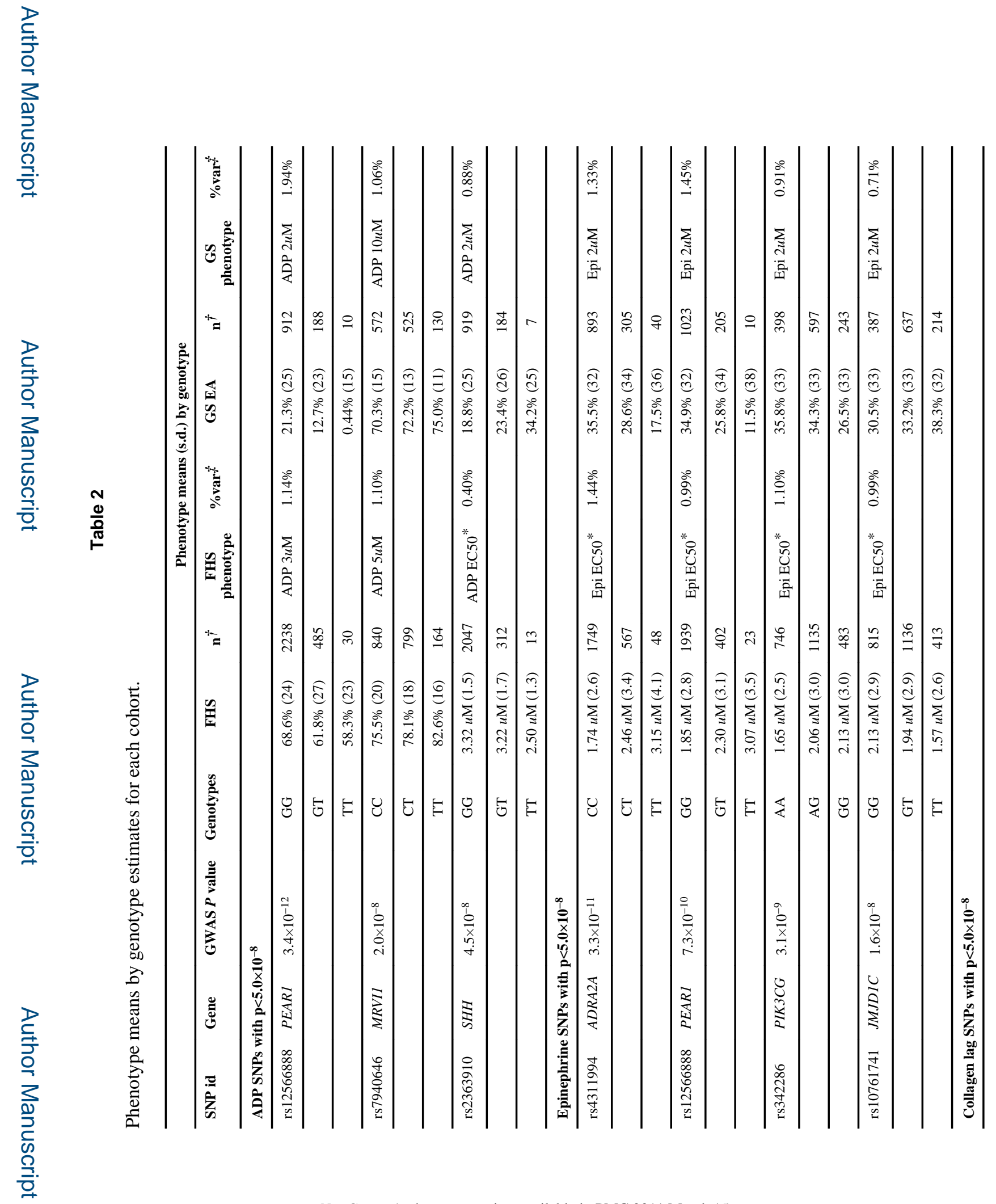

Nat Genet. Author manuscript; available in PMC 2011 March 15. 

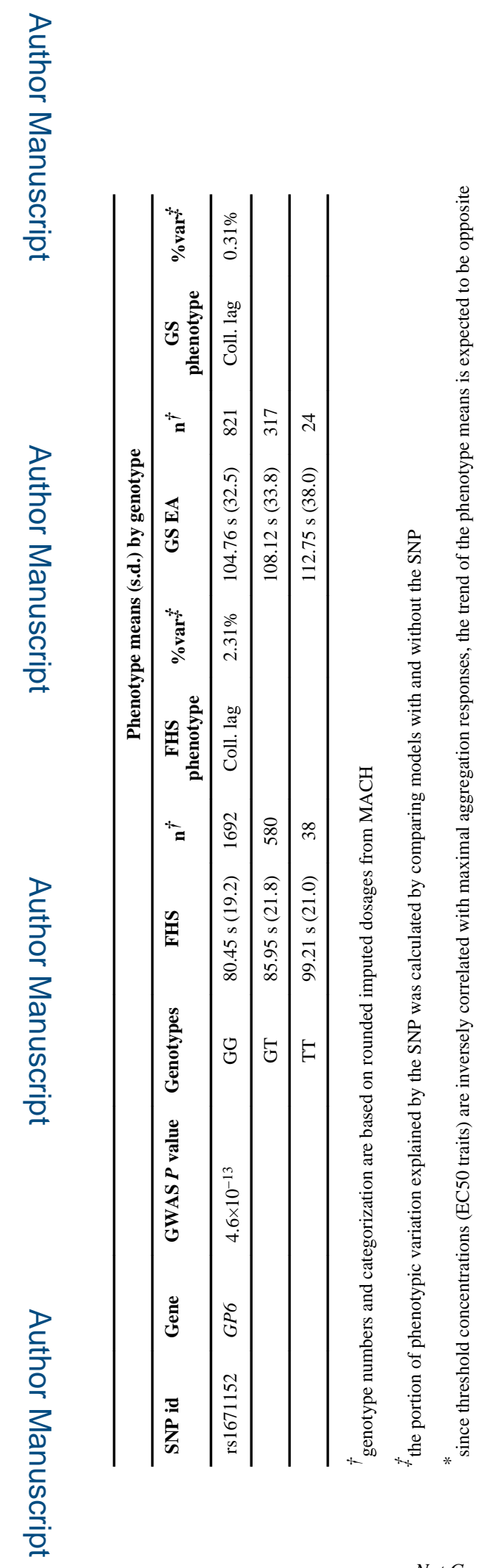

Nat Genet. Author manuscript; available in PMC 2011 March 15 


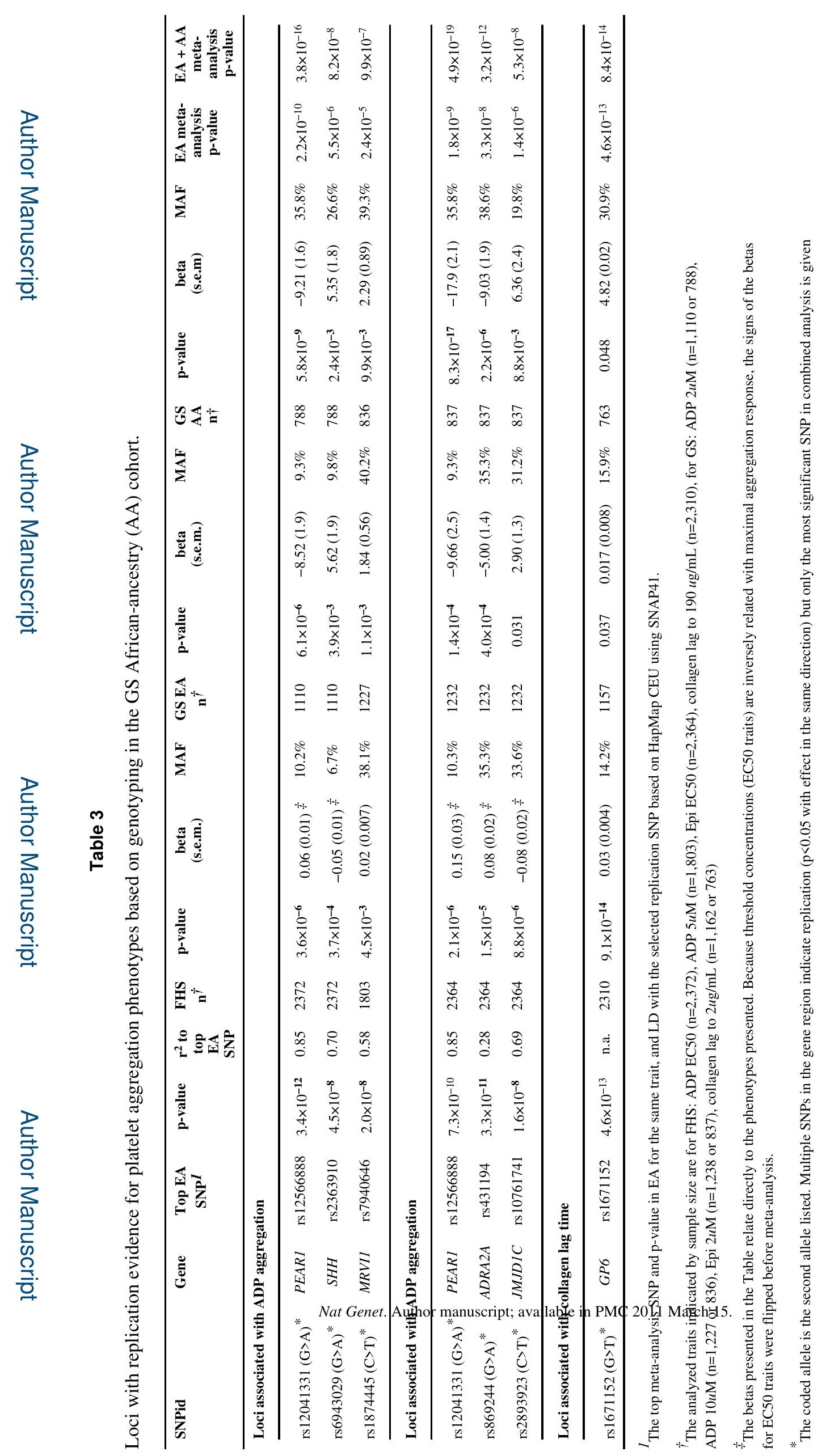




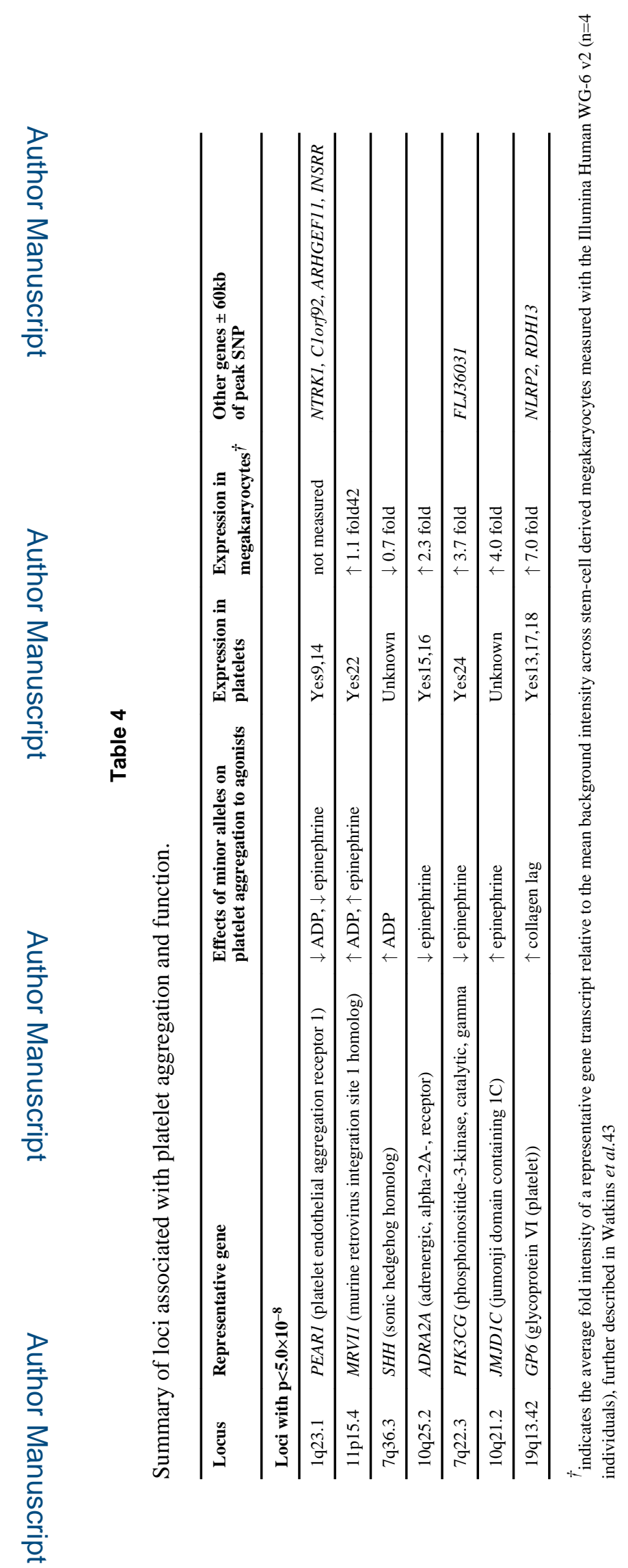

Nat Genet. Author manuscript; available in PMC 2011 March 15. 